\title{
SGLT2 inhibitors and cancer: why further evidence is required
}

\author{
Altamash M. Y. Shaikh ${ }^{1,2,3}$ \\ Received: 6 August 2017 / Accepted: 22 August 2017 / Published online: 13 September 2017 \\ (C) Springer-Verlag GmbH Germany 2017
}

Keywords Bladder · Canagliflozin · Cancer · Dapagliflozin · Empagliflozin · EMPA-REG · Gastrointestinal ·

Meta-analysis · SGLT1 · SGLT2

\section{Abbreviation \\ SGLT2 Sodium-glucose cotransporter 2}

To the Editor: The article on sodium-glucose cotransporter 2 (SGLT2) inhibitors and cancers by Tang et al [1], which was recently published in Diabetologia, is very relevant to practice in today's world. However, this letter is to bring to your kind attention an error in this article. Tang and colleagues conducted a meta-analysis of RCTs to identify any risk of cancer with SGLT2 inhibitors. The results suggested a possible increased risk of bladder cancer with empagliflozin therapy. We noted a possible error in the paper, as discussed below.

The paper quotes ten cases of bladder cancer with empagliflozin, as compared with zero cases with the comparators, and suggests a significant 4.49-fold higher odds of bladder cancer with empagliflozin in a pairwise metaanalysis. The authors state "Most incidences of bladder cancer

Altamash M. Y. Shaikh

ealtamash@gmail.com

1 Department of Medicine, Division of Endocrinology, Saifee Hospital, 15/17, Maharshi Karve Road, Mumbai 400004, India

2 Department of Medicine, Division of Endocrinology, Lokmanya Tilak Municipal Medical College (LTMMC) and Lokmanya Tilak Municipal General Hospital (LTMG), Sion, Mumbai, India

3 Endocrine Clinic, Nagpada, Mumbai, India were identified from the EMPA-REG OUTCOME Trial (empagliflozin: six incidences of bladder cancer, two incidences of bladder transitional cell carcinoma and one incidence of bladder cancer recurrent; placebo: zero incidences)." Following this statement, the authors cite the original paper EMPA-REG OUTCOME Trial publication by Zinman et al [2].

We could not locate the cases of bladder cancer in the EMPA-REG OUTCOME study, in the cited reference [2]. However, this information is available in Table 24 of the US Food and Drug Administration (FDA)-Endocrinologic and Metabolic Drugs Advisory Committee (EMDAC) report [3]. This data suggests that there were four events of bladder cancer in the placebo group, and ten events in the pooled empagliflozin groups. Based on this data, the OR is calculated as 1.25 , with a $p$ value of 0.71 . The four events of bladder cancer in the placebo group are not considered in the analysis by Tang et al [1]. Moreover, a patient-level assessment for causality was not performed by the authors. If we consider this missing data in the overall assessment, the $p$ value for the odds of bladder cancer with empagliflozin remains neutral and non-significant.

Based on the meta-analysis by Tang, et al, canagliflozin may have a protective effect against gastrointestinal cancers with [1]. This may be explained by animal studies, which have shown that, compared to SGLT2, SGLT1 is more involved in glucose uptake by cancer cells and, that, inhibition of this process may have protective effects [4].

On the other hand, in a previously published meta-analysis, an altered risk of bladder cancer was not found with canagliflozin or dapagliglozin. [5]. This study did not include empagliflozin, as only studies prior to 2014 were included. Further, Ptaszynska et al analysed Phase II/III trials for risk of bladder cancer with dapagliglozin (21 studies were included) and did not show causal relationship between dapagliglozin use and bladder cancer [6] 
Hence, based on the available data, the association between SGLT2 inhibitors and risk of cancer in type 2 diabetes needs further investigation.

Duality of interest The author declares that there is no duality of interest associated with this manuscript.

Contribution statement The author was the sole contributor to this paper.

\section{References}

1. Tang H, Dai Q, Shi W, Zhai S, Song Y, Han J (2017) SGLT2 inhibitors and risk of cancer in type 2 diabetes: a systematic review and meta-analysis of randomised controlled trials. Diabetologia. https://doi.org/10.1007/s00125-017-4370-8
2. Zinman B, Wanner C, Lachin JM (2015) Empagliflozin, cardiovascular outcomes, and mortality in type 2 diabetes. NEJM 373:2117-2128

3. US Food and Drug Administration (2016) FDA briefing document: Endocrine and Metabolic Drug Advisory Committee meeting. Available from www.fda.gov/downloads/AdvisoryCommittees/ CommitteesMeetingMaterials/Drugs/Endocrinologicand MetabolicDrugsAdvisoryCommittee/UCM508422.pdf. Accessed 31 August 2017

4. Scafoglio C, Hirayama BA, Kepe Vet al (2015) Functional expression of sodium-glucose transporters in cancer. Proc Natl Acad Sci U S A 112:E4111-E4119

5. Lin H-W, Tseng C-H (2014) A review on the relationship between SGLT2 inhibitors and cancer. Int J Endocrinol. https://doi.org/10. 1155/2014/719578

6. Ptaszynska A, Cohen SM, Messing EM, Reilly TP, Johnsson E, Johnsson K (2015) Assessing bladder cancer risk in type 2 diabetes clinical trials: the dapagliflozin drug development program as a 'case study'. Diabetes Ther 6:357-375 